\title{
Intelligent Analysis Method of Gear Faults Based on FRWT and SVM
}

\author{
Hongfang Chen, Yanqiang Sun, Zhaoyao Shi, and Jiachun Lin \\ Beijing Engineering Research Center of Precision Measurement Technology and Instruments, \\ Beijing University of Technology, Pingleyuan No. 100, Chaoyang District, Beijing, China \\ Correspondence should be addressed to Hongfang Chen; chf0302@126.com
}

Received 5 April 2016; Revised 3 August 2016; Accepted 4 August 2016

Academic Editor: Minvydas Ragulskis

Copyright (C) 2016 Hongfang Chen et al. This is an open access article distributed under the Creative Commons Attribution License, which permits unrestricted use, distribution, and reproduction in any medium, provided the original work is properly cited.

\begin{abstract}
An intelligent analysis method for gear faults based on fractional wavelet transform (FRWT) and support vector machine (SVM) is proposed. Based on this method, FRWT is used to eliminate noise from the gear vibration signal, and the vibration signal after noise elimination is carried thought wavelet packet decomposition and reconstruction. A sequence corresponding to the signal is constructed consisting of the module with the highest level wavelet coefficients after decomposition and feature vectors corresponding to the energy sequence which were obtained by calculation. Then, a particle optimization method is used to optimize SVM parameters, and the feature vectors as training samples are input into SVM for training while the test samples are input for fault recognition. Experimental results show that the gear fault analysis method proposed in this paper is able to effectively extract the weak fault signal. The accuracy rate for identification of the type of gear fault reached $96.7 \%$.
\end{abstract}

\section{Introduction}

As an indispensable component in modern mechanical structure to transmit power and motion, the gear is widely used in various equipment due to its large load capacity, high transmission accuracy, and constant transmission power. As power-transmission equipment develops toward larger scale, greater complexity, automation, and continuous use, gear faults may induce increasingly large losses. Therefore, it is necessary to develop an intelligent gear fault analysis method that can improve the efficiency and accuracy of detection and reduce cost [1-3].

Gear faults are always found in the vibration signal produced by meshing gears. This vibration signal also contains a large number of nonstationary signal components. Using classical spectral analysis methods will result in averaged spectrum values across the whole time scale, which inadvertently conceals some details of mutations. In contrast, a timefrequency method can be used effectively for analysis of nonstationary signals, which can fill the gap in the standard FFTbased method and is developing rapidly as a new approach in the fault analysis field. Wavelet transform (WT) [4-6] is considered as an ideal time-frequency method capable of providing localized information of time and frequency domains at the same time, which can help identify the mutations in the vibration signal. But wavelet transform has limitations, since WT of various scales is similar to a group of frequency-domain band-pass filters [7] which are limited in only time and frequency domain, which makes WT an imperfect choice for chirp type signals whose energy is not optimally gathered in the frequency domain and which exist widely in nature and in various artificial situations. Therefore, analysis methods like fractional Fourier transform (FRFT) [8-10], short-time fractional Fourier transform [11], RadonWigner transform [12], and fractional wavelet transform (FRWT) $[13,14]$ are proposed one after another. FRFT has some unique characteristics but cannot present localized features. Short-time fractional Fourier transform has the fault of low resolution. Radon-Wigner transform is based on quadratic transformations, which have cross terms. FRWT was originally proposed by Mendlovic in 1997, who applied FRFT to the signal to obtain a fractional spectrum, which was then transformed by traditional WT. As a generalized form of WT, FRWT merged many advantages of WT and FRFT 
such as no cross terms, linear characteristics, multiresolution analysis, and fractional domain presentation. Thus, FRWT has attracted more attention as a potential application in signal processing field.

Taking the gear vibration signal as the researched object, two key problems are studied in the practical engineering application, including the gear fault feature extraction and the pattern recognition. An intelligent gear fault analysis method based on a combination of FRWT and SVM is proposed. FRWT makes use of the WT's multiresolution analysis theory and FRFT's time-frequency focusing properties to filter the gear vibration signal. So it can ensure the consistency and effectiveness of time-frequency resolution. Then wavelet packet transform was applied to the denoised signal extracting energy values in the frequency band to build feature vectors, which can be used for training and learning by SVM to further realize intelligent identification of the faulty gear. The SVM method has the advantage of identifying any sample according to the limited samples information. It overcomes the shortcoming of poor classification accuracy caused by insufficient samples in faults of detection of gears. So it improves the efficiency and accuracy of faults of detection of gears.

Combining FRWT and SVM, the method can quickly achieve the detection of finished gears. Then qualities and faults of gears are determined according to the detection results. All in all, this method will provide a powerful analysis means for the diagnosis of gears quality.

\section{Fundamental Principles}

2.1. Fractional Fourier Transform. As a generalized form of Fourier Transform, fractional Fourier transform (FRFT) merges signal information in both the time and frequency domains, which is suitable for time-varying nonstationary signals. The $p$ order fractional Fourier transform of signal $x(t)$ is defined as

$$
X_{\alpha}(u)=\int_{-\infty}^{+\infty} x(t) K_{\alpha}(t, u) d t
$$

Here, $K_{\alpha}(t, u)$ is the transforming kernel function:

$$
\begin{gathered}
\sqrt{\frac{1-j \cot \alpha}{2 \pi}} \exp \left(j \frac{t^{2}+u^{2}}{2} \cot \alpha-t u \csc \alpha\right), \\
\delta \neq n \pi \\
\delta(t-u), \quad \alpha=2 n \pi \\
\delta(t+u), \quad \alpha=(2 n+1) \pi .
\end{gathered}
$$

Here, $p$ is the order of FRFT, which is an arbitrary real number. $\alpha$ represents a rotation angle; $\alpha=p \pi / 2 . n$ is an integer. $\delta(\cdot)$ is an impulse function. The inverse transformation of FRFT is

$$
x(t)=\int_{-\infty}^{+\infty} X(u) K_{-\alpha}(t, u) d u
$$

2.2. Fractional Wavelet Transform. Fractional wavelet transform combines wavelet transform and fractional theory, which promotes multiresolution wavelet analysis theory to a time domain and generalized frequency domain. Meanwhile, it merges characteristics of wavelet transform and fractional theory, which makes it more powerful in signal processing. Mendlovic first proposed the definition of fractional wavelet transform $[13,15]$ and applies it to signal $f(x)$ :

$$
W^{(p)}(a, b)=\iint_{-\infty}^{+\infty} B_{p}\left(x, x^{\prime}\right) f\left(x^{\prime}\right) h_{a b}^{*}(x) d x^{\prime} d x .
$$

Here, $h_{a b}^{*}(x)$ is wavelet basis function and $B_{p}\left(x, x^{\prime}\right)$ is kernel function and can be presented as follows:

$$
\begin{aligned}
B_{p}\left(x, x^{\prime}\right)= & \sqrt{2} \exp \left[-\pi\left(x^{2}+x^{\prime 2}\right)\right] \\
& \cdot \sum_{n=0}^{\infty} \frac{i^{-p n}}{2^{n} n !} H_{n}(\sqrt{2 \pi} x) H_{n}\left(\sqrt{2 \pi} x^{\prime}\right) .
\end{aligned}
$$

Here, $H_{n}$ is $n$ order Hermite polynomials, $\phi=\pi p / 2$, and $p$ is the order of fractional wavelet transform $W^{(p)}(a, b)$, in its value ranging from 0 to 1 .

The reconstruction formula of fractional wavelet transform is

$$
\begin{aligned}
& f(x)=\frac{1}{C} \iiint_{-\infty}^{+\infty} \frac{1}{a^{3}} W^{(p)}(a, b) B_{-p}\left(x, x^{\prime}\right) \\
& \quad \times h\left(\frac{x^{\prime}-b}{a}\right) d a d b d x^{\prime} .
\end{aligned}
$$

We can see that the combination and decomposition of fractional wavelet transform are actually the consistence transform in the time-frequency domain, which accurately regulates information within time domain and frequency domain.

2.3. Support Vector Machine. The advantage of support vector machine (SVM) [16-18] applied to gear vibration signal analysis is that it fits for a small sample of the decision and can maximally excavate the classification knowledge using limited feature information, which has a great practical significance for gear fault analysis. Therefore, we use the SVM in this study to establish the gear fault model by the identification and analysis of the gear fault type from the filtered signal.

The main idea of SVM is to create a hyperplane as a decision interface that has the ability to correctly classify all the training samples and maximize the distance from the nearest point in the training sample to the classification face. The geometry of the hyperplane in the linearly separable two-dimensional input pattern space is shown in Figure 1. Here, the pentacle and rectangles represent the two types of samples, $P$ is the classification lines, and straight lines $P_{1}$ and $P_{2}$ are the nearest samples to the corresponding classification line and parallel to the classification lines. The distance between $P_{1}$ and $P_{2}$ is classification interval. The sample on $P_{1}$ and $P_{2}$ is the SVM. 


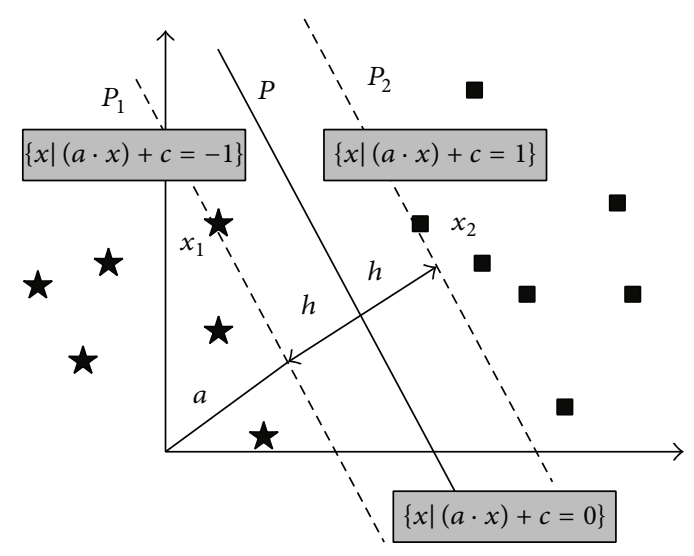

FIGURE 1: Hyperplane in the linearly separable pattern.

Assume that the training sample is $\left\{\left(x_{i}, y_{i}\right)\right\}_{i=1}^{n}$, in which input is $x_{i} \in R^{d}$ and output is $y_{i} \in\{-1,1\}$. The hyperplane equation is $w^{T} x+b=0 . w$ is the adjustable weight vector, $x$ is weight function, and $b$ is the offset. And the constraint condition of the optimization problem is

$$
y_{i}\left(w^{T} x_{i}+b\right) \geq 1, \quad i=1, \ldots, n .
$$

The unknown problem is

$$
\min : \quad \Phi(w, b)=\frac{1}{2}\|w\|^{2} .
$$

This formula is a mathematical inequality problem solved through constrained optimization. The point that makes the equation balance is called support vector. In order to solve the mathematical optimization problem, Lagrange multipliers are introduced to convert it into the dual problem to solve.

Namely, the constraint condition is

$$
\begin{aligned}
\sum_{i=1}^{m} \alpha_{i} y_{i} & =0, \\
\alpha_{i} & \geq 0, \quad i=1,2, \ldots, m .
\end{aligned}
$$

And the unknown problem is

$$
\max : \quad \Phi(\alpha)=\sum_{i=1}^{m} \alpha_{i}-\frac{1}{2} \sum_{i=1}^{m} \sum_{j=1}^{m} \alpha_{i} \alpha_{j} \cdot y_{i} y_{j} x_{i}^{T} y_{j} .
$$

To solve the optimal coefficients above $\widehat{a}, \widehat{w}, \widehat{b}$, the optimal classification function is

$$
f(x)=\operatorname{sgn}\left(\widehat{w}^{T} x+\widehat{b}\right)=\operatorname{sgn}\left(\sum_{i=1}^{n} \widehat{\alpha}_{i} y_{i} x_{i}^{T}+\widehat{b}\right) .
$$

The certain nonlinear mapping can map the sample space into a high-dimensional feature space to convert the liner nonseparable pattern which becomes linearly separable and to construct the optimal classification hyperplane in the high-dimensional feature space. The nonlinear mapping function is called a kernel function. Therefore, the choice of appropriate inner product kernel function can map the low-dimensional to high-dimensional space to achieve linear classification without increasing computational complexity. Compared with linearly separable patterns, the constraints (10) become (parameter $c$ which is used to control the degree of penalty to the wrong sample points)

$$
0 \leq \alpha_{i} \leq c, \quad i=1, \ldots, n .
$$

The optimal classification function becomes

$$
f(x)=\operatorname{sgn}\left(\widehat{w}^{T} x+\widehat{b}\right)=\operatorname{sgn}\left(\sum_{i=1}^{n} \widehat{\alpha}_{i} y_{i} K\left(x_{i}, x\right)+\widehat{b}\right) .
$$

The Gauss radial primary kernel function $K\left(x_{i}, x_{j}\right)=$ $\exp \left(-g\left\|x_{i}-x_{j}\right\|^{2}\right)$ is used in this paper, where $g$ is a constant. As the particle swarm optimization method is faster and more efficient than the grid search method [19], this particle swarm optimization method is used in this paper to optimize the SVM classification model.

The mathematical description of a particle swarm optimization algorithm is as follows: assuming that in a $D$ dimensional optimization space there are a group of particles $m$, where the speed for the $i$ th particle is $v_{i}=\left(v_{i 1}, v_{i 2}, \ldots, v_{i D}\right)$, whose location is $x_{i}=\left(x_{i 1}, x_{i 2}, \ldots, x_{i D}\right)$, and then the optimal position for the $i$ th particle is $p_{i}=\left(p_{i 1}, p_{i 2}, \ldots, p_{i D}\right)$, and the optimal position of the whole population [20] is (the particle update formula is as follows)

$$
\begin{aligned}
v_{i d}(t+1)= & \omega v_{i d}(t)+c_{1} r_{1}\left(p_{i d}(t)-x_{i d}(t)\right) \\
& +c_{2} r_{2}\left(p_{g d}(t)-x_{i d}(t)\right), \\
x_{i d}(t+1)= & x_{i d}(t)+v_{i d}(t+1) .
\end{aligned}
$$

If $v_{i d}>V_{\max }, v_{i d}=V_{\max }$; if $v_{i d}<V_{\max }, v_{i d}=-V_{\max }$, where $i=1,2, \ldots, m$ and $d=1,2, \ldots, D$. $t$ is the number of iterations, $c_{1}, c_{2}$ are accelerating constant, $r_{1}, r_{2}$ are a random number in $[0,1]$, and the $\omega$ is inertia weight.

\section{Intelligent Analysis Method of Gear Fault Detection}

3.1. Noise Removal Based on Fractional Wavelet Transform. Fractional Fourier transform is used to filter the gear vibration signal, whose model is $x(n)=s(n)+d(n)$, where $x(n)$ is the gear vibration signal in the mixed noise, $s(n)$ is a useful component, and $d(n)$ is the background noise. Fractional wavelet transform is applied to both sides of the following equation:

$$
X_{p}\left(v_{k}\right)=S_{p}\left(v_{k}\right)+D_{p}\left(v_{k}\right) .
$$

Here, $X_{p}\left(v_{k}\right), S_{p}\left(v_{k}\right)$, and $D_{p}\left(v_{k}\right)$ are separately the discrete fractional wavelet transform of $x(n), s(n)$, and $d(n)$, and $p$ is the transform order. The basic steps are as follows:

(1) Use a sensor to collect the gear vibration signal $x(n)$ in the acceleration stage, select the range and step order, and apply the fractional Fourier transform to signal 
acquisition, forming a two-dimensional distribution of the signal energy in the fractional Fourier domain $(p, u)$.

(2) In the plane formed in step one, peak points are found through two-dimensional search, where the maximum peaks' corresponding FRFT domain is taken as the best FRFT domain while the optimal order $p_{\text {opt }}$ is determined according to (17), and $p_{\text {opt }}$ order fractional Fourier transform is applied to signal $x(n)$ :

$$
\left(p_{\text {opt }}, u_{\text {opt }}\right)=\underset{p, u}{\arg \max }\left|X_{p}(u)\right|^{2} .
$$

(3) For the signal in the fractional Fourier domain, its wavelet basis function is determined, with proper decomposition layers $N$ selected. After the $N$ th wavelet decomposition, the fractional wavelet signal is obtained, including the Nth low frequency coefficients $c a_{N}$ and high frequency coefficients from 1 st to $N$ th layer $c d_{1}, c d_{2}, \ldots, c d_{N}$.

(4) The signal was decomposed to 1 st to $N$ th fractional wavelet domain signal, where adaptive threshold filter was applied to the corresponding high frequency coefficients (i.e. $c d_{1}, c d_{2}, \ldots, c d_{N}$ ) to eliminate noise.

(5) Wavelet reconstruction was applied to the processed signal, where the basis function and layer number should be ensured the same way as that for wavelet decomposition. Then, wavelet reconstruction is applied to the $N$ layers' frequency coefficients $\left(c a_{N}\right)$ and high frequency coefficients $\left(c d_{1}, c d_{2}, \ldots, c d_{N}\right)$ obtained in the steps above.

(6) $-p_{\text {opt }}$ order fractional Fourier transform was applied to the wavelet reconstruction signal to get the timedomain signal after noise removal.

\subsection{Energy Feature Extraction for Wavelet Packet Frequency} Band. Wavelet packet transform is applied to multiple decompositions and coefficient reconstruction for the filtered signal of the fractional Fourier transform. As the energy projection sequence of gear vibration signal in various frequency bands is a product of the gear transmission mode, analysis of gear vibration signals in different frequency bands can help inconspicuous signal frequency features stand out in a more obvious way as energy change in several subspaces of various resolution. Signal energy after wavelet packet decomposition in each frequency band in various scale spaces is lined up according to their scale as feature vectors. Steps for extracting wavelet packet signal features are as follows:

(1) The $k$-layer wavelet packet and coefficient reconstruction are carried out by filtered vibration signal after FRWT, of which the feature signals $x_{j, m}(i)$ are decomposition coefficients of filtered vibration signal on the $j$ scale: $j=1,2, \ldots k=1,2, \ldots, j m=$ $0,1,2, \ldots, 2^{k-1}$. Here $i$ is the time-domain location; data length of original signal is $N$; data length of feature signal is $n=N / 2^{k}$.
(2) Solve the energy $E_{k m}$ on $k$ th layer within various frequency bands:

$$
E_{k m}=\frac{1}{n-1} \sum_{i=1}^{n}\left|x_{k, m}(i)\right|^{2} \text {. }
$$

(3) Solve the total energy of feature signals. A group of energy sequences $\left\{E_{k m}, m=1,2, \ldots, 2^{k}\right\}$ corresponding to the signal are obtained after wavelet packet decomposition, where the total energy is

$$
E_{t}=\sum_{m=1}^{2^{k}} E_{k m}
$$

(4) Construct the feature vector. As the gear faults will have a great impact on the energy of the signal in each frequency band and $E_{k m}$ may result in some difficulty in data analysis when the value is large, the feature vector is constructed after frequency band energy is normalized:

$$
T=\frac{\left[E_{k 1}, E_{k 2}, \ldots, E_{k 2^{k}}\right]}{E_{t}} .
$$

3.3. Intelligent Recognition for SVM. The normalized feature vectors in Section 3.2 are used as the sample inputs to support vector machines for training and recognition. In this paper, an open source SVM pattern recognition and regression package LIBSVM is used, which utilizes a particle swarm optimization method to optimize the LIBSVM classification model, and then tests the penalty factor $c$ and kernel function parameter $g$ finally obtaining proper SVM correction parameters. The main steps for particle swarm optimization (PSO) to optimize the parameters of SVM for intelligent fault identification are as follows:

(1) Use an initialized particle swarm algorithm, in the search space. Twenty (20) particles are randomly generated, randomly determining each particle's initial position and initial velocity. Initialize error penalty parameters $c$ and Gaussian kernel parameters $g$ of support vector machines, where the optimal ranges of $c$ and $g$ are $[0.1 \sim 100]$ and $[0.1 \sim 1000]$, respectively.

(2) The initialized SVM parameter will be used in the SVM algorithm to build a corresponding model, which is used to predict and classify the test sample. The fitness value of each particle will be calculated according to the fitness function.

(3) The initial particle fitness value is used as the model's initial optimal solution, which is compared with the global optimization objective function value. If the initial fitness value of particles is better than the optimal value of the objective function, the former shall be used as the current optimal objective function value, going on to search for the global optimal solution. 


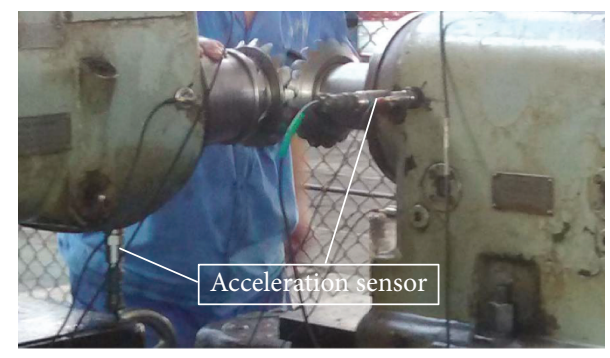

FIGURE 2: Layout of acceleration sensors in the experimental stage.

(4) Update formula of the particle velocity and position is used to update and obtain the best position $p_{\text {best }}$ of each particle itself. After comparing all the best positions of the particles, the best position $g_{\text {best }}$ of all particles is obtained.

(5) Check whether the iteration conforms to the ending condition. If a prescribed error requirement or a certain number of iterations are reached, the iteration will be stopped. Otherwise, go back to step (2).

(6) The penalty parameter $c$ and Gauss kernel parameter $g$ obtained through optimization are used as SVM parameters. Input training samples for training and respective category labels are established according to different types of gear faults. Meanwhile, "one against rest" SVC is used for training to ultimately obtain some certain training parameter information (including support Vector, pull-down factor, and network bias.).

(7) The test samples are input into support vector machines for recognition, outputting the test results, which are used to determine gear fault types.

\section{Experiments}

In this paper, the rolling-gear inspection machine is used to carry out the experiment, in which the arrangement of acceleration sensor used to collect gear vibration signal is shown in Figure 2. The gear drive is a one-level drive, whose driving wheel and a driven wheel share a gear tooth number of 17 , with a transmission ratio of $1: 1$. The driving wheel rotation speed is $750-1000 \mathrm{rpm}$. The sampling frequency of the vibration signal sound is $10 \mathrm{kHz}$. The gear vibration signals were collected in three cases of normal gear, shaft unbalance fault, and pitting fault.

When gears are in states of normal meshing, shaft unbalance fault, and pitting fault (Figure 3), respectively, curves of measured vibration signal in the time domain and frequency domain are shown in Figures 4(a), 4(b), and 4(c) separately, where obvious modulation phenomena occur in the time-domain signals for all of three types of gears mentioned above. From the FFT frequency spectrum diagram in Figure 3, background noise has masked the noise generated by gear meshing, which makes the time and frequencydomain curves of the three types of gears so similar that

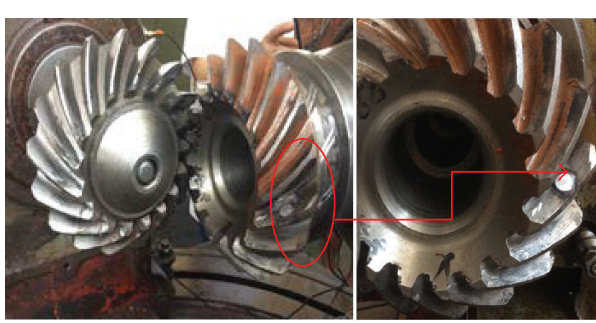

FIgURE 3: Tooth surface pitting.

it is difficult to distinguish between them based only on a comparison of the curves in either the time domain or in the frequency domain.

A gear fault analysis method based on a combination of FRWT and SVM proposed in this paper is used to analyze the three kinds of gear vibration signals mentioned in Figure 4.

Step 1. Fractional wavelet transform is applied to the collected gear vibration signal to eliminate background noise, extracting the useful signal. Fractional Fourier transform is applied to the gear vibration signal with a transform order of p. According to the peak search and calculation, an optimal order can be determined, by the basis on which a narrowband filter is constructed to mask the optimal FRFT domain signal and further to eliminate the energy of background noise while retaining that of useful vibration signal. Therefore, the gear vibration signal after denoising based on the fractional wavelet transform is obtained. Figure 5 is the 2-dimensional distribution of signal energy before filtering and after narrow band filtering in the fractional Fourier transform domain in three cases, showing that the filtering method proposed in this article can achieve better noise suppression effects.

Step 2. Fractional Fourier transform is applied to the signal filtering to get useful gear vibration signal $W(t)$, which is decomposed and reconstructed by db6 3-layer wavelet packet and to obtain the decomposition energy spectrum. Frequency of gear vibration signal ranges from 0 to $2000 \mathrm{~Hz}$, and sampling frequency $f_{s}$ is $10 \mathrm{kHz}$. Therefore, when $k$-layer wavelet packet decomposition is applied to the gear vibration signal, the node bandwidth at the $k$ th layer is

$$
f_{\mathrm{BW}}=\frac{f_{s}}{2^{k+1}}
$$

where frequency range for each node is

$$
m f_{\mathrm{BW}} \sim(m+1) f_{\mathrm{BW}}, \quad m=0,1,2, \ldots, 2^{k}-1 .
$$

Here, $m$ represents the node number at the $k$ th layer in wavelet decomposition. Wavelet packet frequency band and frequency correspondence are shown in Table 1.

A sequence corresponding to the signal which consists of the module of the highest level wavelet coefficients after decomposition and feature vectors corresponding to the 

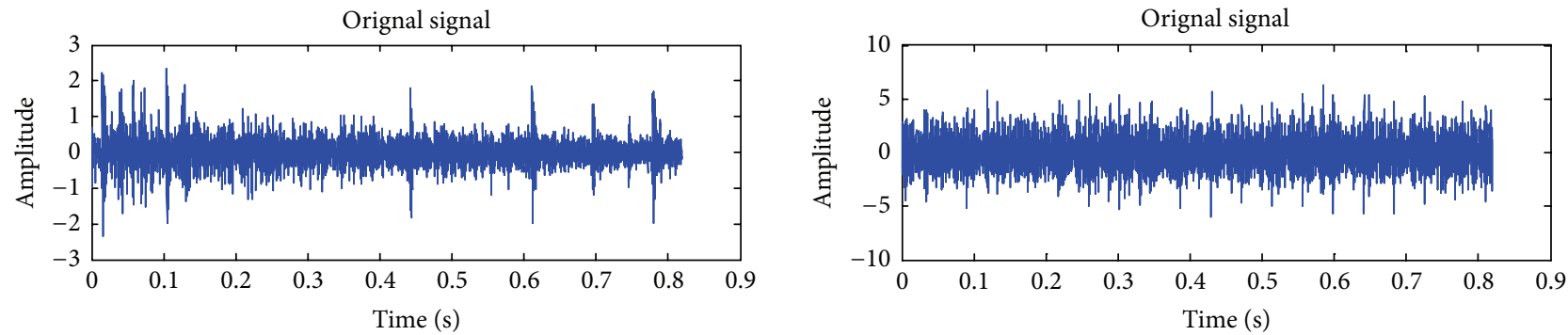

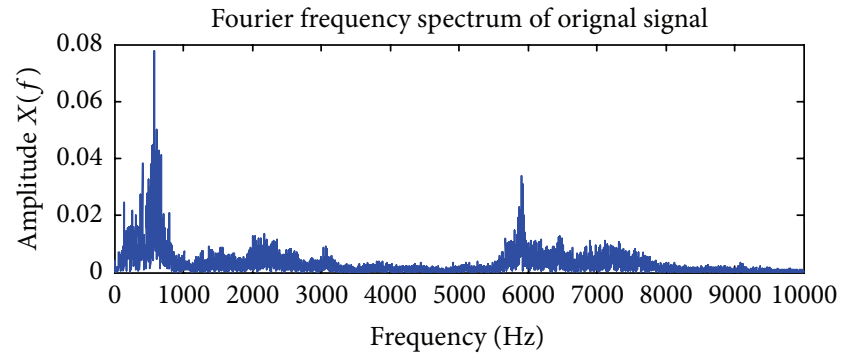

(a) Gear vibration signal of normal gear meshing

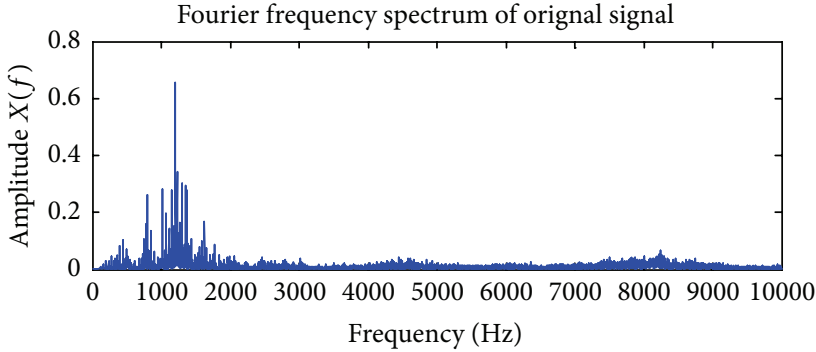

(b) Gear vibration signal of shaft unbalance fault
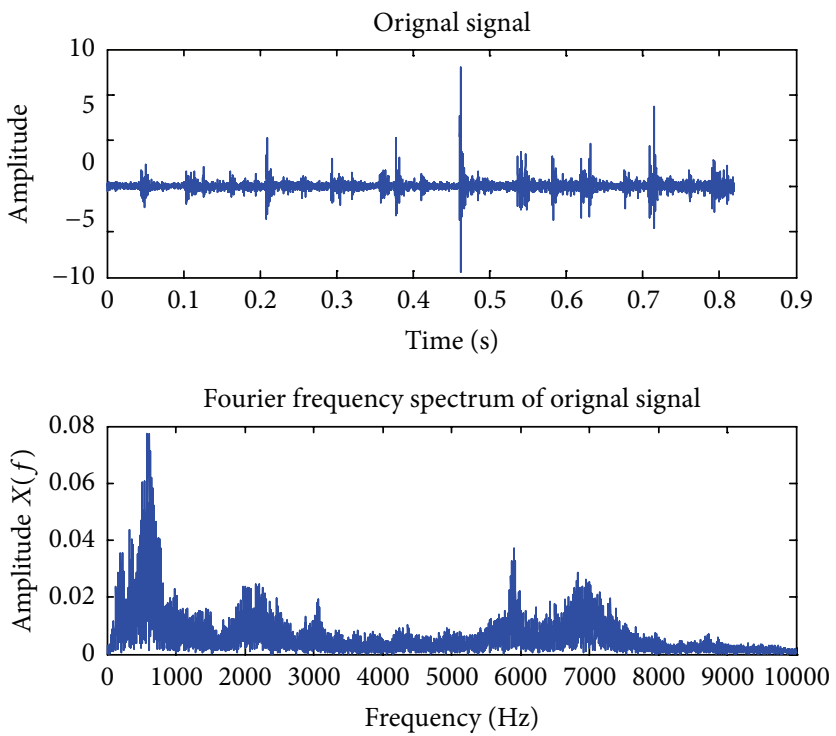

(c) Gear vibration signal of pitting fault

FIGURE 4: Gear vibration signal.

energy sequence were obtained according to (20). Figure 6 shows the histogram for the feature vectors of the three cases of normal gear meshing, shaft unbalance fault, and pitting fault.

As is shown in Figure 6, in vibration signal wavelet energy spectrum for different types of gear fault, energy is concentrated mainly within the 1st band because of the vibration due to stiffness stimulation and load variation. When the gear fault exists, the amplitude of the meshing frequency and its frequency band tend to produce large growth. However, it is difficult to directly observe the effects of background noise in the FFT spectrum.
As can be seen clearly in the wavelet energy spectrum diagram, the characteristic value of the 1st frequency band for shaft unbalance gear fault is $2 \%$ lower than that of normal gear. At the same time, the characteristic value of the 3 rd frequency band is increased by about $90 \%$ and the characteristic value of the 6th frequency band is decreased by about $99 \%$.

As for the pitting gear fault, the characteristic value in the 1 st band decreases by $29 \%$ compared with the normal gear operation. Meanwhile, the characteristic value of the 3rd frequency band is increased by about $97 \%$ and the characteristic value of the 6th frequency band is increased by 
Before filtering
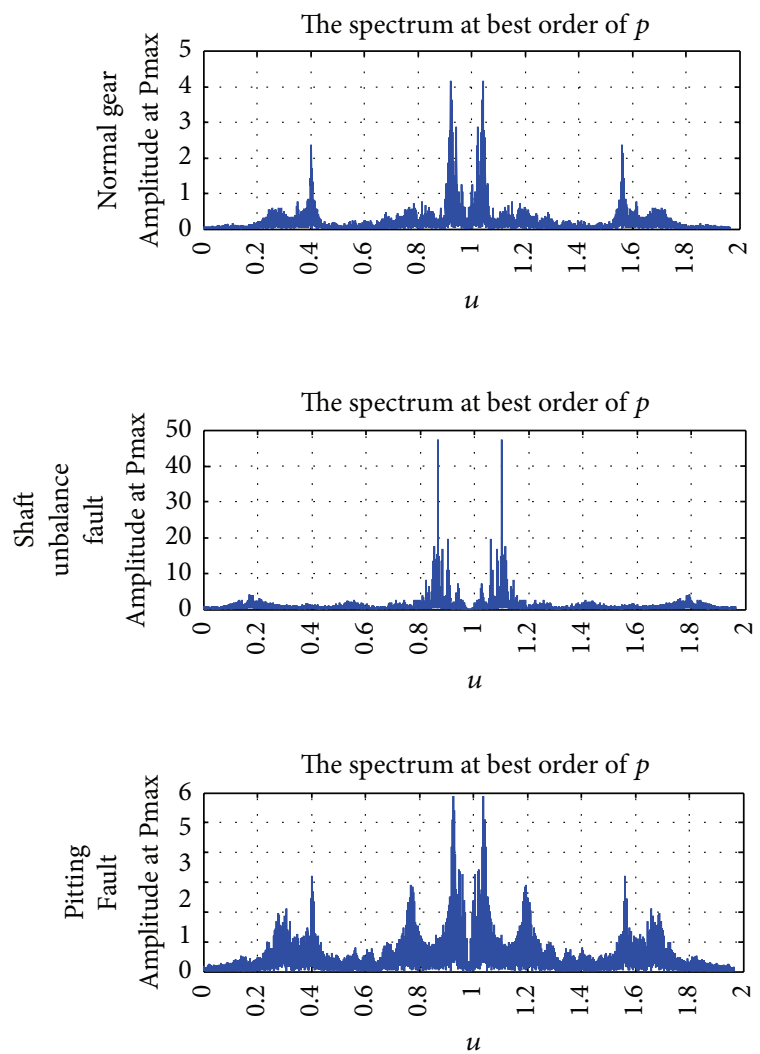

After filtering
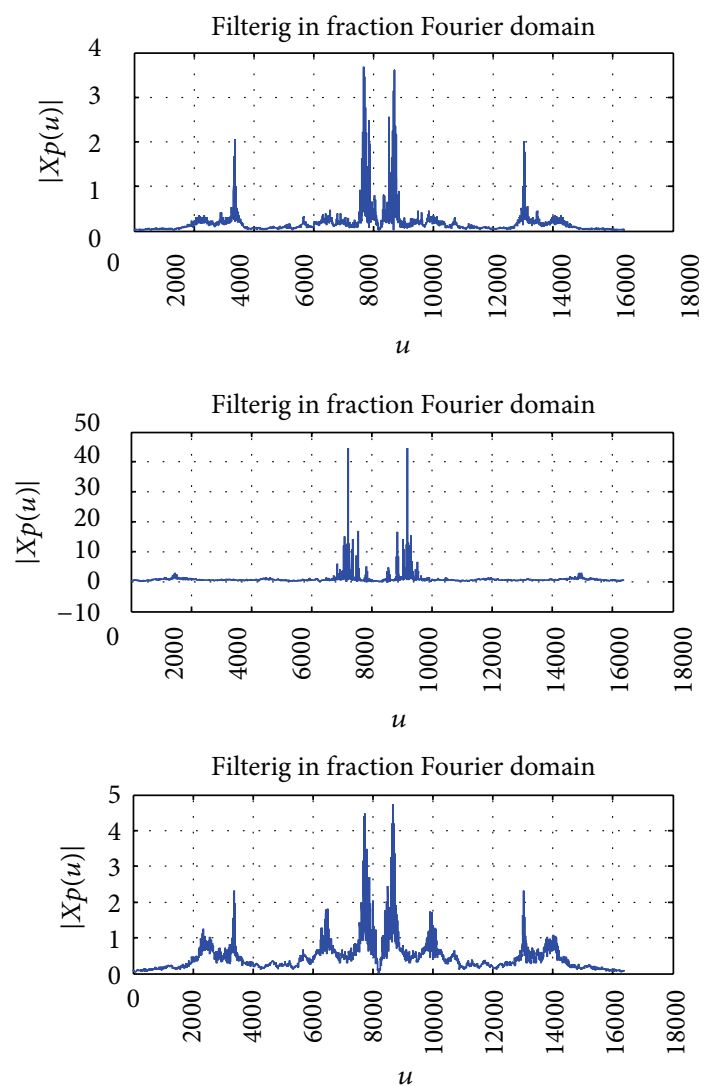

FIGURE 5: 2-dimensional distribution of signal energy before filtering and after narrow band filtering on fractional Fourier transform domain in three cases.

TABLE 1: A wavelet packet frequency band-frequency tablet.

\begin{tabular}{lcc}
\hline Band serial number & Sample point & Frequency range $(\mathrm{Hz})$ \\
\hline 1 & $W(3,0)$ & $0 \sim 625$ \\
2 & $W(3,1)$ & $625 \sim 1250$ \\
3 & $W(3,2)$ & $1250 \sim 1875$ \\
4 & $W(3,3)$ & $1875 \sim 2500$ \\
5 & $W(3,4)$ & $2500 \sim 3125$ \\
6 & $W(3,5)$ & $3125 \sim 3750$ \\
7 & $W(3,6)$ & $3750 \sim 4375$ \\
8 & $W(3,7)$ & $4375 \sim 5000$ \\
\hline
\end{tabular}

about $40 \%$. The characteristic values of the other bands also appear to increase in varying degrees.

The above analysis shows that there are marked differences in characteristic values of the three cases of normal gear, shaft unbalance fault, and pitting fault, which can be used as feature vectors for gear fault type recognition.

Step 3. Use SVM to study the feature vectors of multiple sets of gear vibration signal data. The sample consists of 60 groups of gears, where the 20 sets of gear vibration signal samples are collected, respectively, in three cases of normal gear, shaft unbalance fault, and pitting fault. The 60 sets of feature vectors are obtained by calculation, according to first and second steps of the mentioned method. Divide the feature vectors into two groups, training samples and test samples, each containing 10 groups of normal gears, 10 groups of shaft unbalance fault gears, and 10 groups of pitting fault gears. The training samples are optimized by the particle optimization method, and the optimization results are shown in Figure 7.

After the completion of training, test samples are input into SVM for recognition, the results of which are shown in Figure 8. In Figure 8, vertical coordinates 1, 2, and 3 represent the normal gear, shaft unbalance fault, and pitting fault, respectively. The circle represents the actual test set classification of the input data while the asterisk represents predictive test set classification of the input data. As is directly shown, the results of practical training and prediction for three kinds of gear fault type using multiclassification SVM can reach an accuracy of $96.7 \%$.

Experimental results prove that application of the fractional Fourier transform proposed in this paper can effectively extract useful signals reflecting the fault feature under 


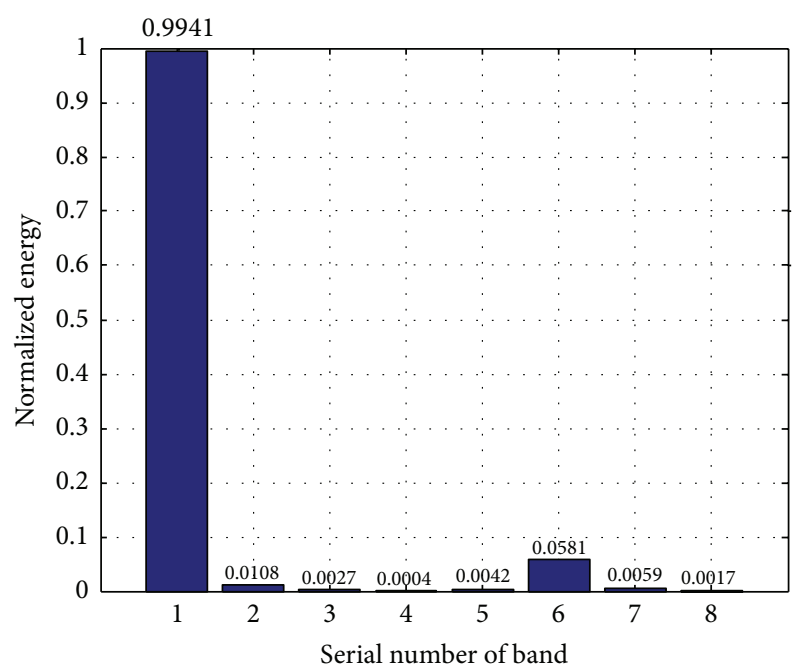

(a) Normal gear

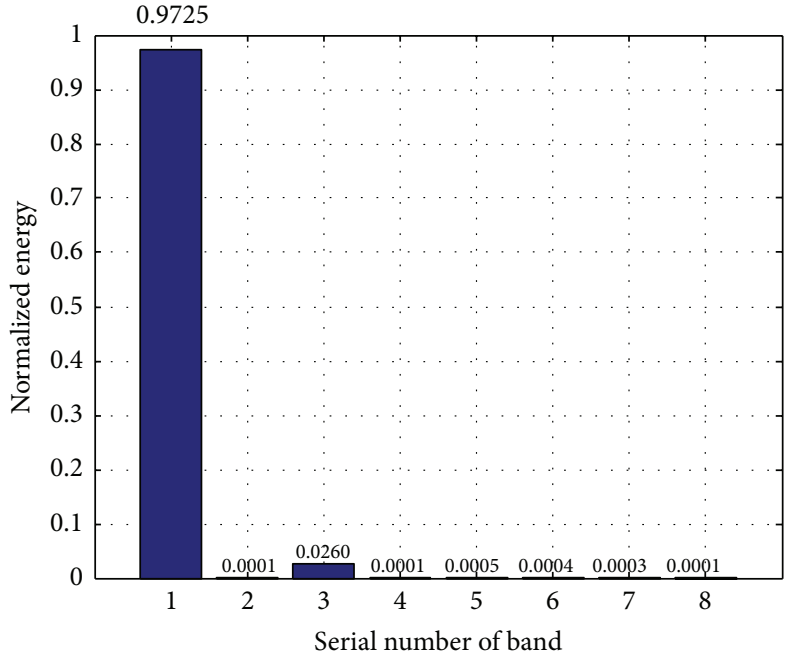

(b) Shaft unbalance fault

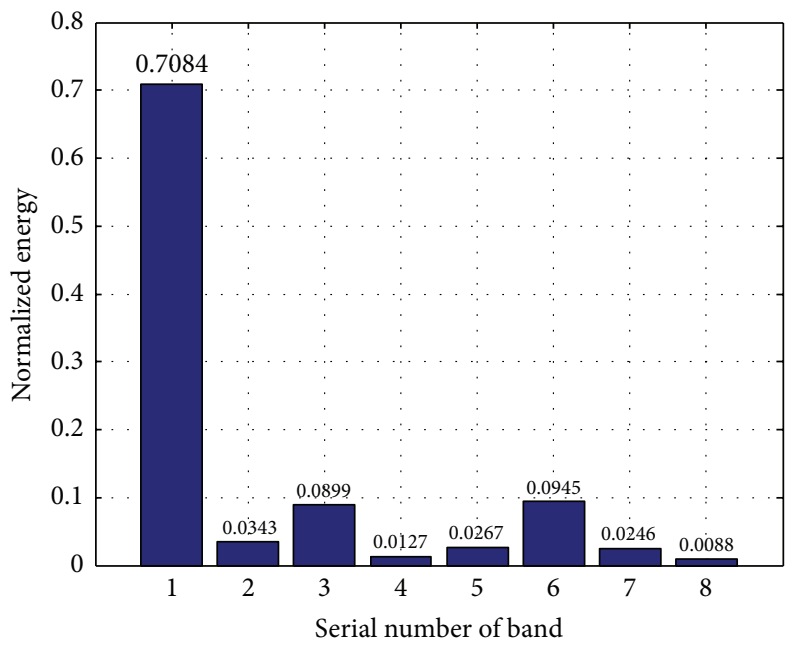

(c) Pitting fault

FIGURE 6: Histogram of the feature vector.

a background condition of powerful noise. SVM can intelligently recognize gear fault types without the artificial manipulation.

\section{Conclusions}

This paper studies a gear fault detection method based on the feature vector of gear vibration signals. For the collected vibration signal, the noise is eliminated by the FRWT method. Using the wavelet packet transform, the feature vectors are constituted by the frequency band energy. On this basis, the feature vectors are trained and classified by support vector machines. Through the experiments, the FRWT can extract the useful signals which reflect the gear fault features, achieving good effects in elimination of background noise. And the automatic recognition of gear fault is realized through the clustering and classification of vibration signals vector by SVM, so that it can solve the problem of faults recognition in time and frequency domain which is caused by the interference of the background noise and the complexity of the side band. Therefore, it is intelligent, efficient, and accurate for the gear vibration analysis method based on FRWT and SVM.

\section{Competing Interests}

The authors declare that they have no competing interests.

\section{Acknowledgments}

The research is supported by national major scientific instruments and equipment development projects (No. 2013YQ17053904), national science and technology major 


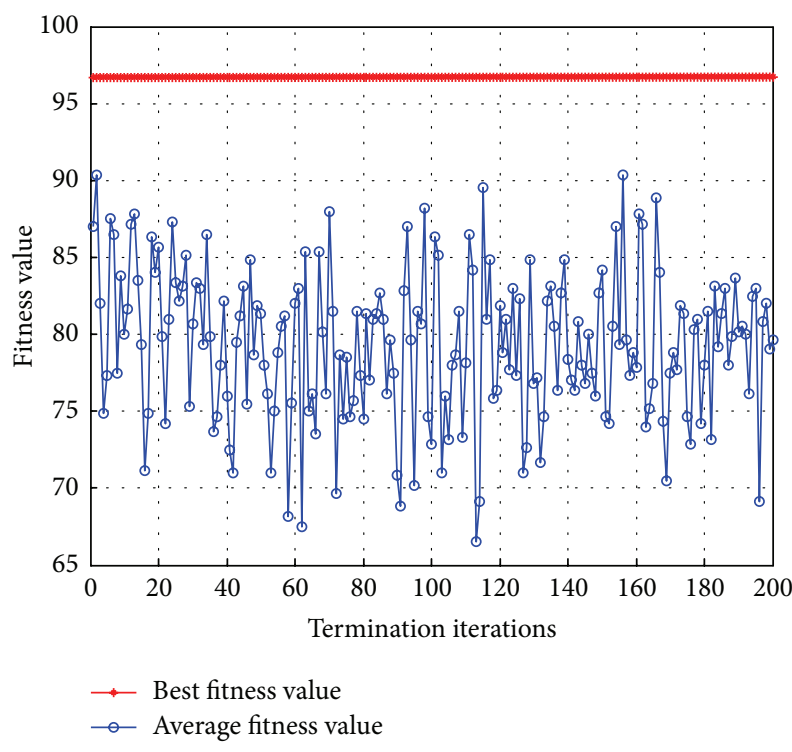

FIGURE 7: Optimal results of SVM via particle optimal method.

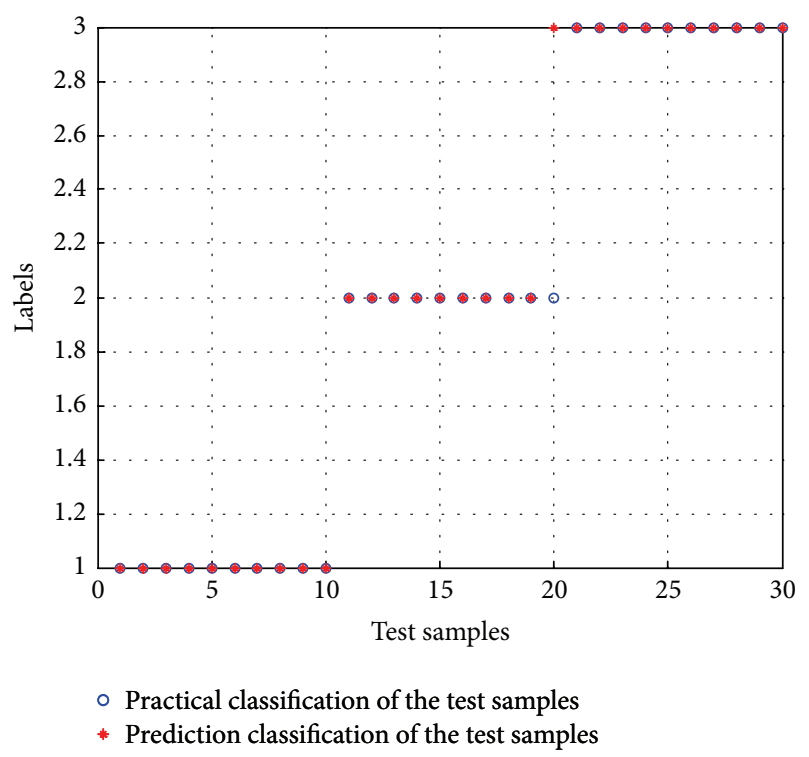

FIGURE 8: Recognized results of gear fault.

special projects (No. 2010ZX04014-091), and project of national science foundation of china (No. 50805003).

\section{References}

[1] M. Amarnath and I. R. Praveen Krishna, "Empirical mode decomposition of acoustic signals for diagnosis of faults in gears and rolling element bearings," IET Science, Measurement \& Technology, vol. 6, no. 4, pp. 279-287, 2012.

[2] Z. Y. Shi, H. Lin, and J. Lin, "Current status and trends of large gears metrology," Journal of Mechanical Engineering, vol. 49, no. 10, pp. 35-44, 2013.

[3] B. Samanta, "Artificial neural networks and genetic algorithms for gear fault detection," Mechanical Systems and Signal Processing, vol. 18, no. 5, pp. 1273-1282, 2004.
[4] R. Bajric, N. Zuber, G. A. Skrimpas, and N. Mijatovic, "Feature extraction using discrete wavelet transform for gear fault diagnosis of wind turbine gearbox," Shock and Vibration, vol. 2016, Article ID 6748469, 10 pages, 2016.

[5] S. G. Mallat, "A theory for multiresolution signal decomposition: the wavelet representation," IEEE Transactions on Pattern Analysis and Machine Intelligence, vol. 11, no. 7, pp. 674-693, 1989.

[6] Z. K. Peng and F. L. Chu, "Application of the wavelet transform in machine condition monitoring and fault diagnostics: a review with bibliography," Mechanical Systems and Signal Processing, vol. 18, no. 2, pp. 199-221, 2004.

[7] C. Gargour, M. Gabrea, V. Ramachandran, and J.-M. Lina, "A short introduction to wavelets and their applications," IEEE Circuits and Systems Magazine, vol. 9, no. 2, pp. 57-68, 2009.

[8] L. B. Almeida, "The fractional fourier transform and timefrequency representations," IEEE Transactions on Signal Processing, vol. 42, no. 11, pp. 3084-3091, 1994.

[9] L. Yuan, "Wavelet-fractional Fourier transforms," Chinese Physics B, vol. 17, no. 1, pp. 170-176, 2008.

[10] V. A. Narayanan and K. M. M. Prabhu, “The fractional Fourier transform: theory, implementation and error analysis," Microprocessors and Microsystems, vol. 27, no. 10, pp. 511-521, 2003.

[11] R. Tao, Y.-L. Li, and Y. Wang, "Short-time fractional Fourier transform and its applications," IEEE Transactions on Signal Processing, vol. 58, no. 5, pp. 2568-2580, 2010.

[12] J. C. Wood and D. T. Barry, "Linear signal synthesis using the Radon-Wigner transform," IEEE Transactions on Signal Processing, vol. 42, no. 8, pp. 2105-2111, 1994.

[13] D. Mendlovic, Z. Zalevsky, D. Mas, J. García, and C. Ferreira, "Fractional wavelet transform," Applied Optics, vol. 36, no. 20, pp. 4801-4806, 1997.

[14] L. Chen and D. Zhao, "Optical image encryption based on fractional wavelet transform," Optics Communications, vol. 254, no. 4-6, pp. 361-367, 2005.

[15] Y. Huang and B. Suter, "The fractional wave packet transform," Multidimensional Systems and Signal Processing, vol. 9, no. 4, pp. 399-402, 1998.

[16] D. Wang, K.-L. Tsui, P. W. Tse, and M. J. Zuo, "Principal components of superhigh-dimensional statistical features and support vector machine for improving identification accuracies of different gear crack levels under different working conditions," Shock and Vibration, vol. 2015, Article ID 420168, 14 pages, 2015.

[17] A. Widodo and B.-S. Yang, "Support vector machine in machine condition monitoring and fault diagnosis," Mechanical Systems and Signal Processing, vol. 21, no. 6, pp. 2560-2574, 2007.

[18] B. Samanta, K. R. Al-Balushi, and S. A. Al-Araimi, "Artificial neural networks and support vector machines with genetic algorithm for bearing fault detection," Engineering Applications of Artificial Intelligence, vol. 16, no. 7-8, pp. 657-665, 2003.

[19] R. Eberhart and J. Kennedy, "New optimizer using particle swarm theory," in Proceedings of the 6th International Symposium on Micro Machine and Human Science, pp. 39-43, Nagoya, Japan, October 1995.

[20] J. Kennedy and R. Eberhart, "Particle swarm optimization," in Proceedings of the IEEE International Conference on Neural Networks, pp. 1942-1948, Perth, Ausralia, November-December 1995. 


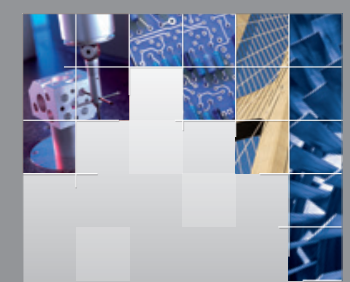

\section{Enfincering}
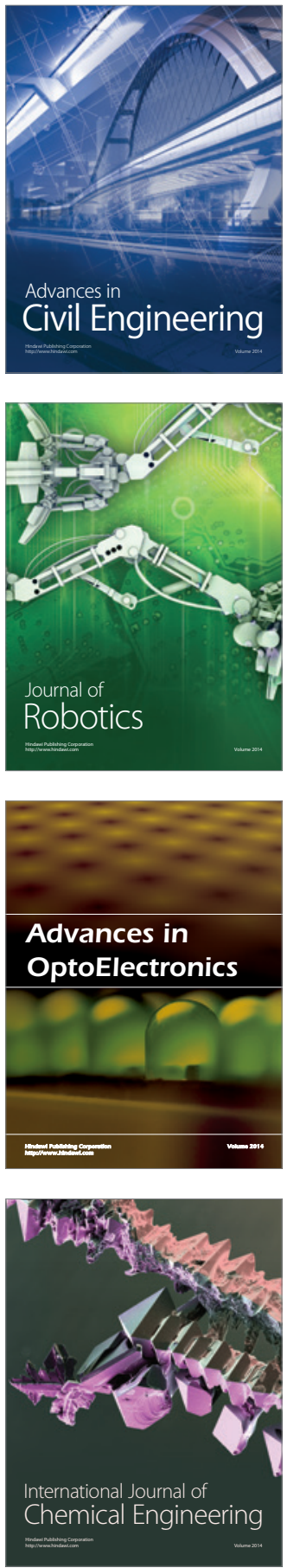

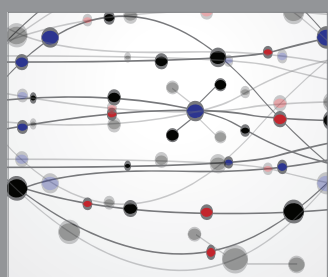

The Scientific World Journal

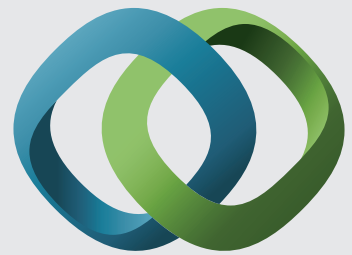

\section{Hindawi}

Submit your manuscripts at

http://www.hindawi.com
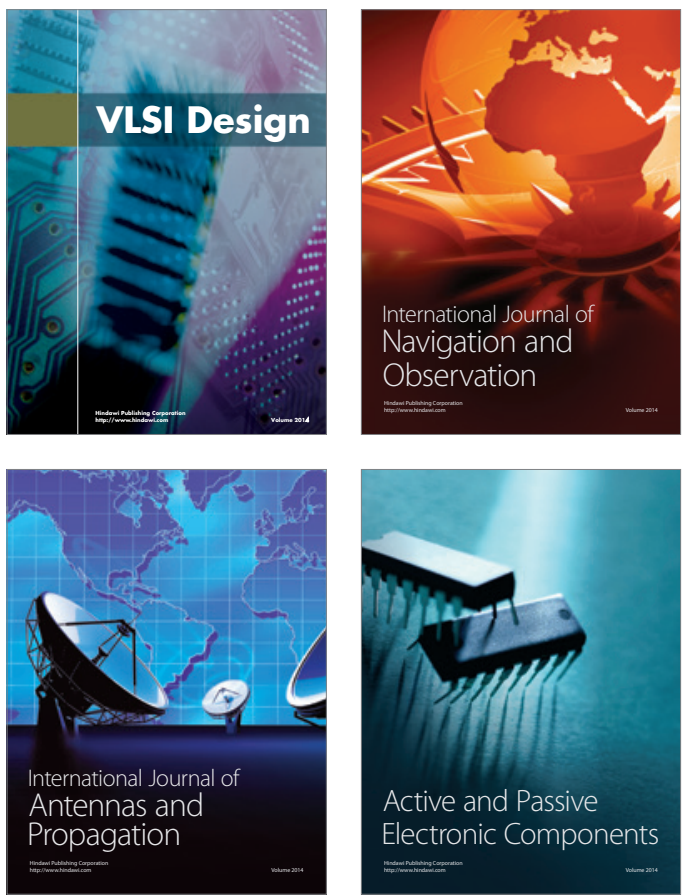
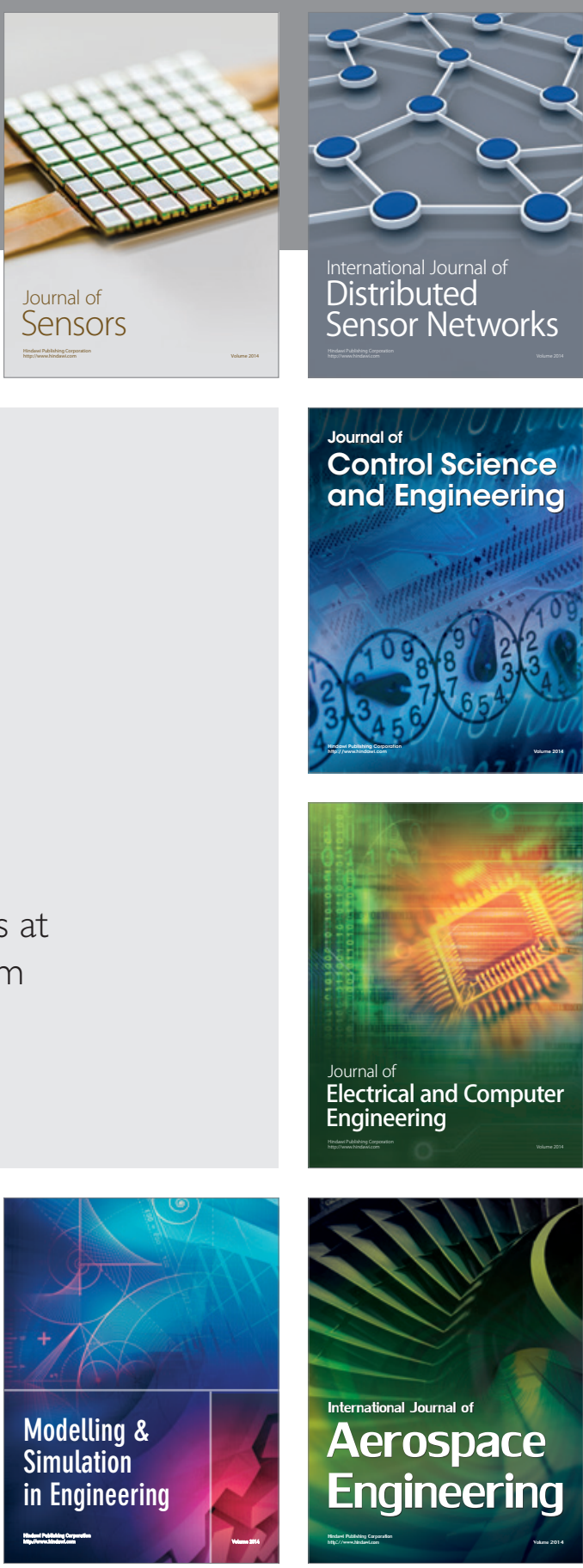

International Journal of

Distributed

Sensor Networks

Journal of

Control Science

and Engineering
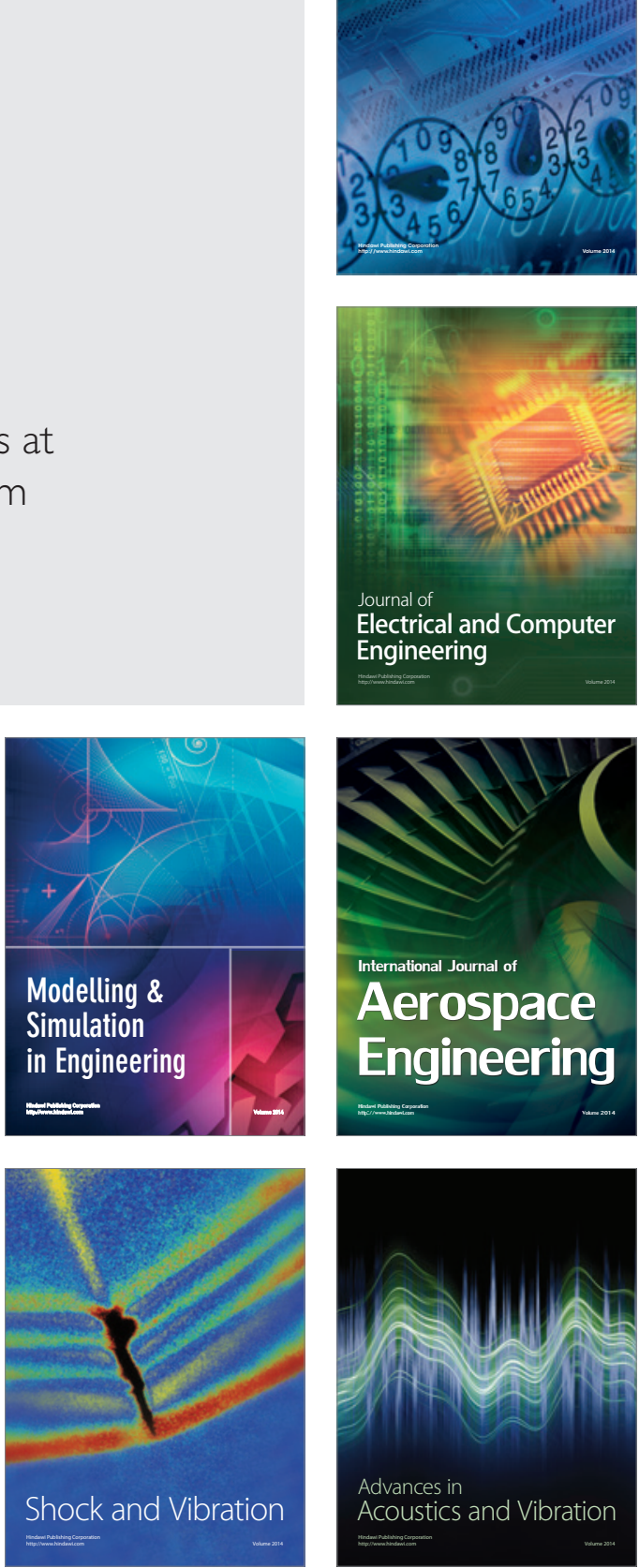